\title{
SATISFAÇÃO NO TRABALHO DOS BANCÁRIOS E SEUS FATORES DETERMINANTES - UMA REVISÃO INTEGRATIVA
}

\section{PREVALENT FACTORS OF BANKING PROFESSIONALS' JOB SATISFACTION: AN INTEGRATIVE REVIEW}

\author{
Rosangela Correa \\ Escola de Enfermagem de Ribeirão Preto - USP \\ rc.sp@usp.br
}

Silvia Helena Henriques Camelo

Escola de Enfermagem de Ribeirão Preto - USP

shcamelo@eerp.usp.br

Laura Andrian Leal

Escola de Enfermagem de Ribeirão Preto - USP

laura.andrian.leal@usp.br

Submissão: 08/08/2016

Aprovação: 17/10/2017

\section{RESUMO}

O estudo teve o objetivo de identificar a presença de satisfação no trabalho dos profissionais do setor bancário e o papel da gerência dos serviços. Trata-se de uma revisão integrativa e a coleta de dados foi realizada em quatro bases eletrônicas, nos anos de 2015 e 2016 . Foram selecionados 21 artigos publicados no período de 2010 a 2016. Os resultados mostraram índices diferenciados de satisfação no trabalho dos profissionais do setor bancário. Dentre os fatores positivos que podem interferir nessa satisfação estão: liderança participativa, ambiente de trabalho saudável e de aprendizagem, treinamento, comprometimento organizacional, entre outros. Conclui-se que os resultados deste estudo possam contribuir para a reflexão dos profissionais das instituições financeiras, bem como gestores da área de saúde do trabalhador, quanto à necessidade de promoção da satisfação no trabalho dos funcionários, considerando que esta pode influenciar potencialmente o ambiente organizacional e trazer consequências para as metas ou resultados esperados pela organização.

Palavras-Chave: Satisfação no emprego. Condições de trabalho. Administração financeira. Ambiente de trabalho. Setor público. 


\begin{abstract}
The study aimed to identify the presence of satisfaction in the work of banking professionals and the role of service management. This is an integrative review and data collection was conducted in four electronic databases, in the years of 2015 and 2016. 21 articles published between 2010 to 2016 were selected. The results showed differentiated indices of satisfaction in the work of the professionals of the banking sector; The positive factors that may interfere in this satisfaction are: participative leadership, a healthy workplace and corporative training, organizational commitment, among others. It is concluded that the results of this study can contribute to the reflection of the professionals of the financial institutions, as well as managers of the worker's health area, regarding the need to promote satisfaction in the work of employees, considering that this can potentially influence the organizational environment and bring consequences to the goals or results expected by the organization.
\end{abstract}

Keywords: Job Satisfaction. Working Conditions. Financial Management. Work Environment. Public Sector. 


\section{INTRODUÇÃO}

$\mathrm{Na}$ atualidade, no cenário mundial, a competitividade entre as instituições tem sido destacada, levando as empresas a se depararem com grandes desafios no que diz respeito à gestão financeira e de pessoas.

A fim de manter-se no mercado de forma atuante, faz-se de suma importância que as práticas de gestão de pessoas nas organizações sejam aprimoradas, diante de maiores exigências relacionadas à qualificação dos funcionários, da constante diminuição dos postos de trabalho e da precarização das relações e condições trabalhistas (SEVERINO; EID; CHIARELLO, 2013).

Sabe-se que o trabalho, em suas diferentes formas, pode repercutir na saúde do trabalhador de forma satisfatória ou insatisfatória. Como resultado satisfatório, evidencia-se a realização do indivíduo a partir da criação de coisas e de si mesmo, produzindo uma sensação de bem-estar físico e emocional, além de explorar as potencialidades humanas. Contudo, dependendo das condições deste trabalho, ele pode representar insatisfação, sofrimento, desvalorização, desgaste físico e estresse emocional (LUNARDI FILHO, 1995; MINAYO; ASSIS; OLIVEIRA, 2011; NEVES; SELIGMANN, 2006). Nesse sentido, as organizações devem ficar atentas com seu capital humano proporcionando um ambiente laboral saudável.

A satisfação no trabalho pode ser definida como um vínculo afetivo ou emocional, relacionado a várias facetas do trabalho de um indivíduo. É o estado emocional positivo resultante do trabalho do empregado ou da experiência prática, resultando na satisfação com o trabalho (HAREEM, 2013). A satisfação teria como consequências, comportamentos favoráveis à empresa, tais como a redução da taxa de rotatividade e da ausência no trabalho, bem como o aumento de produtividade, demonstrando que o vínculo afetivo do indivíduo com o trabalho traz importantes resultados para a organização, além de ganhos na vida dos indivíduos (SIQUEIRA, 2008).

Nas organizações financeiras, com destaque para o setor bancário, a competitividade e a produtividade surgem de forma impactante, modificando o ambiente de trabalho. Por isso a satisfação no ambiente laboral tem um papel relevante e pode influenciar, de forma positiva, a organização de todo o processo de trabalho (GAGANDEEP, 2015). Em relação a isso, vale ressaltar que a categoria bancária está entre as que mais sofrem com doenças ocupacionais relacionadas à forma de gestão, trabalhando sob pressão, por maior produtividade e enfrentando sentimentos de frustração, insegurança e pressão psicológica (SILVA, NAVARRO, 2012). Exige-se do trabalhador bancário um amplo conhecimento do mercado financeiro, domínio de tecnologia, habilidade no relacionamento com os clientes e equipe de vendas, além de maior capacidade de decisão (MAXIMO et al., 2011).

A transformação no processo de trabalho dos bancários acarretou também profunda mudança no status dessa função, depreciando essa categoria profissional, antes encarada como símbolo de prestígio, sendo que aquele que ingressava no banco permanecia nessa carreira até a aposentadoria. Diferentemente, na atualidade, essa categoria vem sendo substituída por jovens que desejam apenas financiar seus estudos, não vislumbrando mais a carreira bancária como promissora (BARRETO, 2015). Vale destacar, contudo, que essa realidade pode variar entre os países, tendo como exemplo a Índia, onde as pessoas ainda consideram o trabalho em banco como símbolo de status social (GAGANDEEP, 2015).

Estudos têm revelado, de um lado, que as alterações no ritmo e na natureza do trabalho bancário têm provocado alto nível de estresse, sobrecarga de trabalho, problemas como o esgotamento, tensões, sedentarismo e doenças crônicas. Diante desses desgastes, as vítimas de estresse no trabalho mostram falta de confiança, baixa motivação no local de trabalho, 
aumento da pressão arterial, insatisfação no trabalho e intenção de deixar o trabalho, aumentando assim os índices de rotatividade da organização (GEORGE; ZAKKARIYA, 2015; PETARLI et al., 2015; SILVA; BARRETO, 2012; SILVA; NAVARRO, 2012).

De outro lado, a literatura aponta que o trabalhador satisfeito encontra motivação para desenvolver sua aprendizagem, responder positivamente ao trabalho e aumentar seu desempenho (SARAVANI; ABBASI, 2013). Além disso, a literatura tem evidenciado que fatores como bom ambiente local de trabalho, supervisão eficaz realizada pela chefia, cooperação dos colegas, ausência de discriminação no trabalho, aceitação empregado, segurança no trabalho e remuneração são importantes determinantes da satisfação no trabalho bancário (GAGANDEEP, 2015; KAUR, 2015).

Ainda, há os estudos que identificam práticas de qualidade de vida, manutenção do clima organizacional e fatores que contribuem ou interferem na satisfação no seu trabalho. Alguns desses analisam os índices de satisfação em diversos segmentos de trabalhadores (ESTIVALETE; ANDRADE, 2012; GIRONDI, 2007; MACHADO; JAROUJ, 2007; SILVA; LIMA, 2007; VALLE, 2007).

Nessa perspectiva, considerando o contexto descrito, este estudo apresenta os seguintes questionamentos: Existe satisfação no trabalho dos profissionais que atuam em serviços de instituições financeiras, especificamente do setor bancário? Que fatores gerenciais interferem na satisfação no trabalho desses profissionais?

Com esta pesquisa espera-se provocar a reflexão dos profissionais do setor financeiro, quanto ao seu preparo para atuar no setor, bem como dos gestores de pessoas sobre o seu papel na administração e valorização desses funcionários, aliando satisfação no trabalho e produtividade no alcance dos objetivos da empresa. Os resultados poderão, ainda, contribuir para a elaboração de estratégias para a promoção da satisfação no trabalho, com efeitos positivos para a saúde do trabalhador.

Nesse sentido, este estudo teve o objetivo de identificar, por meio da produção científica, a presença de satisfação no trabalho dos profissionais do setor bancário e o papel da gerência dos serviços na satisfação dos funcionários.

$\mathrm{O}$ artigo foi estruturado em seis seções, incluindo esta introdução: inicialmente aprofundamos a temática da satisfação no trabalho; posteriormente descrevemos a metodologia utilizada para a coleta de dados; a seguir apresentamos os resultados encontrados, caracterizando os artigos selecionados; a discussão dos dados e as considerações finais dos autores fecham o texto.

\section{SATISFAÇÃO NO TRABALHO}

Os primeiros estudos sobre Satisfação no Trabalho ocorreram por volta de 1912, a partir das pesquisas de Taylor sobre Gerência Científica, nas quais o conceito de satisfação ligava-se intimamente à questão da fadiga e do salário e suas respectivas interferências na produtividade (CURA; RODRIGUES, 1999). Posteriormente, entre 1970-1980, a satisfação no trabalho foi concebida como uma atitude individual, preditora de comportamentos como a redução da rotatividade e absenteísmo, melhor produtividade e desempenho (SIQUEIRA, 2008).

Nessa perspectiva, Locke $(1969,1976)$ define satisfação no trabalho como o estado emocional agradável resultante da avaliação positiva e subjetiva do indivíduo sobre seu trabalho, obtendo a realização de seus valores pessoais relacionados às condições de trabalho (ambiente de trabalho, o trabalho em si, condições de remuneração, reconhecimento e 
promoções), aos agentes de trabalho (colegas, supervisores e subordinados), necessitando de constantes análises individuais, considerando o componente cognitivo que envolve a racionalidade do indivíduo sobre o trabalho e o componente emocional, relacionado a como a pessoa se sente no trabalho (LOCKE, 1969; LOCKE, 1976).

Assim, a satisfação no trabalho passa a ser compreendida como um resultado do ambiente organizacional sobre a saúde do trabalhador. Seria um sentimento diante das políticas e práticas gerenciais, percepção de justiça e retorno pelo trabalho, suporte organizacional, como também a reciprocidade da empresa em relação aos esforços e investimentos aplicados na organização (SIQUEIRA, 2008).

Dessa forma, pode-se dizer que o trabalho prejudicado por fatores estressantes ou de insatisfação, está associado com a piora da saúde física e mental, mesmo quando há outros fatores controlados, dentre eles, os fatores demográficos, os hábitos de saúde e a renda. Indivíduos mais satisfeitos com seu trabalho apresentam menor ocorrência de doenças físicas ou mentais (FLETCHER; SINDELAR; YAMAGUCHI, 2011).

No que diz respeito ao trabalho em instituições financeiras, as novas tecnologias adotadas, de um lado, otimizaram o trabalho bancário, aumentando a produtividade e a eficiência dos serviços prestados, bem como a lucratividade do setor. Mas de outro lado, elas provocaram aumento da intensidade do trabalho, o que alterou as condições do mesmo e repercutiu na saúde dos bancários (NAVARRO; CARRIJO, 2009).

Nesse sentido, é importante que a organização adote instrumentos que possibilitem gestão eficaz de pessoas, com avaliação da satisfação, visando à promoção da saúde e da qualidade de vida no trabalho. Neste contexto, observa-se que as empresas têm buscado programas que promovam a satisfação de seus trabalhadores, percebendo-se também um crescente interesse do meio acadêmico em pesquisar temas relativos à satisfação no trabalho, pelo fato de que empregados satisfeitos imprimem maior qualidade a suas atividades, produzindo mais e aumentando a competitividade da organização (RUEDA, 2015).

\section{METODOLOGIA}

Este estudo é uma revisão integrativa, que se trata de ampla abordagem metodológica referente às revisões, permitindo a inclusão de estudos experimentais ou não, combinando dados da literatura teórica e empírica (WHITTEMORE; KNALF, 2005).

O método da revisão integrativa permite a síntese de múltiplos estudos já publicados, auxiliando na obtenção de conclusões gerais a respeito de uma área de estudo. Inicia-se pela determinação do objetivo específico a ser atingido, na direção dos questionamentos a serem pesquisados, e busca em bases de dados de trabalhos sobre a temática, obedecendo-se aos critérios de inclusão já estabelecidos. Os dados são categorizados, interpretados e sintetizados, produzindo assim, um saber uniforme e embasado do assunto pesquisado (MENDES; SILVEIRA; GALVÃO, 2008).

A revisão integrativa permite ainda, a inclusão de estudos experimentais e nãoexperimentais para uma compreensão completa do fenômeno analisado. Pode combinar dados da literatura teórica e empírica, além de incorporar a definição de conceitos, revisão de teorias e/ou a análise de problemas metodológicos, proporcionando a síntese do conhecimento e a incorporação da aplicabilidade de resultados significativos na prática (SOUZA; SILVA; CARVALHO, 2010).

A estratégia de busca utilizada foi a consulta às bases eletrônicas Medical Literature Analysis and Retrieval System Online-Medline; Literatura Latino-Americana e do Caribe em 
Ciências da Saúde - Lilacs; SCOPUS Database; The Cochrane Library e Scientific Eletronic Library Online - SCIELO, sendo a coleta de dados realizada entre junho de 2015 a junho de 2016.

Para o levantamento bibliográfico dos artigos, utilizamos os Descritores em Ciências da Saúde (Decs): satisfação no emprego, condições de trabalho, administração financeira, ambiente de trabalho, setor público. Foram pesquisadas também as seguintes palavras-chaves: setor bancário, desempenho profissional, bancário, compromisso organizacional, respectivamente também em inglês e espanhol. O boleano utilizado na busca foi: and.

Os critérios usados para a seleção dos artigos foram: artigos científicos publicados em periódicos nacionais e internacionais, em português, inglês e espanhol, nos últimos seis anos, nas bases de dados selecionadas, que abordassem a temática investigada, respondendo aos objetivos dessa pesquisa. No período da coleta de dados os estudos estavam disponíveis na íntegra nas bases de dados consultadas. $\mathrm{O}$ acesso às bases de dados foi realizado em uma Universidade do Estado de São Paulo. Foram excluídos trabalhos como teses, dissertações, livros e capítulos de livros com o objetivo de eliminar publicações que não passaram por rigorosa avaliação e revisão, de modo a selecionar apenas a literatura indexada.

\section{RESULTADOS}

\subsection{Características dos artigos}

Foram encontrados 39 artigos, correspondendo aos descritores e palavras-chave utilizados na busca. Destes, foram selecionados 23 artigos, sendo 19 desses $(82,6 \%)$ em inglês e quatro (17,3\%) em português, todos publicados entre 2010 e 2016, conforme Quadro 1.

\section{Quadro 1 - Distribuição dos artigos relacionados à satisfação no trabalho de bancários,} segundo título, autores, ano e periódico de publicação

(continua...)

\begin{tabular}{|c|c|c|c|c|}
\hline Título & Autores & Ano & Periódico & $\begin{array}{l}\text { Base de } \\
\text { ados }\end{array}$ \\
\hline $\begin{array}{l}\text { Comparison of Factors } \\
\text { Influencing Job Satisfaction in } \\
\text { Domestic and Foreign Banks in } \\
\text { China }\end{array}$ & Xiaoyan; Puxi & 2010 & IEE Xplore Digital Library & $\begin{array}{l}\text { SCOPUS } \\
\text { Database }\end{array}$ \\
\hline $\begin{array}{l}\text { Relationships between } \\
\text { organizational support, customer } \\
\text { orientation, and work outcomes A } \\
\text { study of frontline bank employees }\end{array}$ & Yavas; Babacus & 2010 & $\begin{array}{l}\text { International Journal of } \\
\text { Bank Marketing }\end{array}$ & $\begin{array}{l}\text { SCOPUS } \\
\text { Database }\end{array}$ \\
\hline $\begin{array}{lcc}\text { A } & \text { Multilevel Model } & \text { Of } \\
\text { Organizational Commitment } & \end{array}$ & Armutlulu; Noyan & 2011 & $\begin{array}{l}\text { Procedia }- \text { Social and } \\
\text { Behavioral Sciences }\end{array}$ & $\begin{array}{l}\text { SCOPUS } \\
\text { Database }\end{array}$ \\
\hline $\begin{array}{l}\text { Contingency approach to strategic } \\
\text { management: a test of the } \\
\text { mediating effect of leader } \\
\text { member exchange on the } \\
\text { relationship between } \\
\text { psychological empowerment and } \\
\text { job satisfaction in } 21 \mathrm{st} \text { century }\end{array}$ & Bitmis; Ergeneli & 2011 & $\begin{array}{l}\text { Procedia Social } \\
\text { Behavioral Sciences }\end{array}$ & SCOPUS \\
\hline
\end{tabular}




\begin{tabular}{|c|c|c|c|c|}
\hline workplace & & & & Database \\
\hline $\begin{array}{l}\text { Job Satisfaction in Private } \\
\text { Banking Sector of Pakistan }\end{array}$ & Hassan, et. al. & 2011 & $\begin{array}{l}\text { Global Journal of } \\
\text { Management and Business } \\
\text { Research }\end{array}$ & $\begin{array}{l}\text { SCOPUS } \\
\text { Database }\end{array}$ \\
\hline $\begin{array}{l}\text { Research on the Relations } \\
\text { between } \\
\text { Managerial Self-efficacy and Job } \\
\text { Satisfaction }\end{array}$ & Kai; Yuyang & 2011 & $\begin{array}{l}\text { National Natural Foundation } \\
\text { of China }\end{array}$ & $\begin{array}{l}\text { SCOPUS } \\
\text { Database }\end{array}$ \\
\hline $\begin{array}{l}\text { Impacto do treinamento como } \\
\text { variável preditora da satisfação } \\
\text { com o trabalho }\end{array}$ & Santos; Mourão & 2011 & USP -R. Adm. São Paulo & $\begin{array}{l}\text { SCOPUS } \\
\text { Database }\end{array}$ \\
\hline $\begin{array}{l}\text { Trabalhadores Afastados por } \\
\text { Transtornos Mentais e de } \\
\text { Comportamento: o Retorno ao } \\
\text { Ambiente de Trabalho e suas } \\
\text { Consequências na Vida Laboral e } \\
\text { Pessoal de Alguns Bancários }\end{array}$ & $\begin{array}{l}\text { Olivier; } \\
\text { Behr }\end{array}$ & 2011 & RAC, Curitiba & $\begin{array}{l}\text { SCOPUS } \\
\text { Database }\end{array}$ \\
\hline
\end{tabular}

\section{Quadro 1 - Distribuição dos artigos relacionados à satisfação no trabalho de bancários,} segundo título, autores, ano e periódico de publicação

(continua...)

\begin{tabular}{|c|c|c|c|c|}
\hline $\begin{array}{l}\text { A Comparative Study of } \\
\text { Organizational Climate and Job } \\
\text { Satisfaction } \\
\text { in Public, Private and Foreign } \\
\text { Banks }\end{array}$ & Bhutto, et. al. & 2012 & $\begin{array}{l}\text { Canadian Center of Science } \\
\text { and Education }\end{array}$ & $\begin{array}{l}\text { SCOPUS } \\
\text { Database }\end{array}$ \\
\hline $\begin{array}{l}\text { Employment Security as } \\
\text { Moderator on the Effect of Job } \\
\text { Security on } \\
\text { Worker's Job Satisfaction and } \\
\text { Well Being }\end{array}$ & Fatimah, et. al. & 2012 & $\begin{array}{l}\text { Canadian Center of Science } \\
\text { and Education }\end{array}$ & $\begin{array}{l}\text { SCOPUS } \\
\text { Database }\end{array}$ \\
\hline $\begin{array}{l}\text { Information control, role } \\
\text { perceptions, and work outcomes } \\
\text { of boundary-spanning } \\
\text { frontline managers }\end{array}$ & Rigopoulou, et. al. & 2012 & Journalof Business Research & $\begin{array}{l}\text { SCOPUS } \\
\text { Database }\end{array}$ \\
\hline $\begin{array}{l}\text { An Empirical Study on the } \\
\text { Relationship } \\
\text { Detween } \\
\text { and Job Satisfaction Itself among } \\
\text { the Employeers of Both Public } \\
\text { and Private Sector Banks of } \\
\text { Guntur Urban Region }\end{array}$ & Reddy; Gummadi & 2013 & $\begin{array}{l}\text { Internacional }- \text { Business } \\
\text { Management }- \text { Medwell } \\
\text { Journals }\end{array}$ & $\begin{array}{l}\text { SCOPUS } \\
\text { Database }\end{array}$ \\
\hline $\begin{array}{l}\text { Determinants of job satisfaction } \\
\text { in the banking sector: the case }\end{array}$ & Tlaiss & 2013 & $\begin{array}{l}\text { EmeraldGroupPublishingLi } \\
\text { mited }\end{array}$ & $\begin{array}{l}\text { SCOPUS } \\
\text { Database }\end{array}$ \\
\hline
\end{tabular}




\begin{tabular}{|c|c|c|c|c|}
\hline ofLebanese managers & & & & \\
\hline $\begin{array}{l}\text { Investigating the influence of job } \\
\text { rotation on performance by } \\
\text { considering skill variation and job } \\
\text { satisfaction of bank employees }\end{array}$ & Saravani; Abbasi & 2013 & TechnicalGazette & $\begin{array}{l}\text { SCOPUS } \\
\text { Database }\end{array}$ \\
\hline $\begin{array}{l}\text { Neuroticism as a Determinant of } \\
\text { Job Satisfaction among Bank } \\
\text { Employees }\end{array}$ & $\begin{array}{l}\text { Hlatywayo; } \\
\text { Mhlanga; Zingwe }\end{array}$ & 2013 & $\begin{array}{l}\text { Mediterranean Journal of } \\
\text { Social Sciences }\end{array}$ & $\begin{array}{l}\text { SCOPUS } \\
\text { Database }\end{array}$ \\
\hline $\begin{array}{l}\text { The role of employee job } \\
\text { satisfaction in strengthening } \\
\text { customer repurchase intentions }\end{array}$ & Gounaris; Boukis & 2013 & $\begin{array}{l}\text { Journal of } \quad \text { Services } \\
\text { Marketing }\end{array}$ & $\begin{array}{l}\text { SCOPUS } \\
\text { Database }\end{array}$ \\
\hline $\begin{array}{l}\text { Perception of bank employees' } \\
\text { towards working environment of } \\
\text { selected Indian universal banks }\end{array}$ & Gagandeep & 2015 & $\begin{array}{l}\text { International Journal of } \\
\text { Bank Marketing }\end{array}$ & $\begin{array}{l}\text { SCOPUS } \\
\text { Database }\end{array}$ \\
\hline
\end{tabular}




\section{Quadro 1 - Distribuição dos artigos relacionados à satisfação no trabalho de bancários, segundo título, autores, ano e periódico de publicação}

\begin{tabular}{|c|c|c|c|c|}
\hline $\begin{array}{l}\text { A Influência do Desenvolvimento } \\
\text { Profissional e da Identificação } \\
\text { Organizacional na Satisfação no } \\
\text { Trabalho }\end{array}$ & $\begin{array}{l}\text { Mourão; } \\
\text { Monteiro; Viana }\end{array}$ & 2014 & Psico & $\begin{array}{l}\text { Literatura } \\
\text { Latino- } \\
\text { Americana } \\
\text { e do } \\
\text { Caribe em } \\
\text { Ciências } \\
\text { da Saúde - } \\
\text { Lilacs; }\end{array}$ \\
\hline $\begin{array}{l}\text { Job related stress and job } \\
\text { satisfaction: a comparative study } \\
\text { among bank employees }\end{array}$ & $\begin{array}{l}\text { George; } \\
\text { Zakkariya }\end{array}$ & 2015 & $\begin{array}{l}\text { Journal of Management } \\
\text { Development }\end{array}$ & $\begin{array}{l}\text { SCOPUS } \\
\text { Database }\end{array}$ \\
\hline $\begin{array}{l}\text { Autoavaliação do estado de saúde } \\
\text { e fatores associados: um estudo } \\
\text { em trabalhadores bancários. }\end{array}$ & Petarli, et. al. & 2015 & Cad. Saúde Pública & $\begin{array}{l}\text { Literatura } \\
\text { Latino- } \\
\text { Americana } \\
\text { e do } \\
\text { Caribe em } \\
\text { Ciências } \\
\text { da Saúde - } \\
\text { Lilacs }\end{array}$ \\
\hline $\begin{array}{l}\text { Exploring the moderating role of } \\
\text { context satisfaction between job } \\
\text { characteristics and turnover } \\
\text { intention of employees of Indian } \\
\text { public sector banks }\end{array}$ & Piyali, et. al. & 2015 & $\begin{array}{l}\text { Journal of Management } \\
\text { Development }\end{array}$ & $\begin{array}{l}\text { SCOPUS } \\
\text { Database }\end{array}$ \\
\hline $\begin{array}{l}\text { An Analysis of Employee } \\
\text { Satisfaction of Private Banks in } \\
\text { Myanmar. }\end{array}$ & Myint, et. al. & 2016 & $\begin{array}{l}\text { International } \\
\text { Management }\end{array}$ & $\begin{array}{l}\text { The } \\
\text { Cochrane } \\
\text { Library }\end{array}$ \\
\hline $\begin{array}{l}\text { The effect of employee CSR } \\
\text { attitudes on job satisfaction and } \\
\text { organizational commitment: } \\
\text { evidence from the Bangladeshi } \\
\text { banking industry }\end{array}$ & $\begin{array}{l}\text { Shafiqur; Debbie; } \\
\text { Mehrdokht }\end{array}$ & 2016 & Responsibility & $\begin{array}{l}\text { SCOPUS } \\
\text { Database }\end{array}$ \\
\hline
\end{tabular}

Fonte: Elaborado pelos autores.

Em relação aos delineamentos da pesquisa, 21 estudos utilizaram a abordagem metodológica quantitativa, um utilizou a abordagem quali-quantitativa e, outro, a abordagem qualitativa. O estudo que utilizou abordagem qualitativa foi do tipo descritivo e exploratório. O estudo quali-quantitativo utilizou o método hierárquico (nested); e dentre os quantitativos, os métodos são do tipo descritivo, retrospectivo, exploratório, comparativo, observacional e transversal. Nesse sentido, o objeto de pesquisa pode ser analisado por diferentes delineamentos metodológicos.

Após a coleta de dados foi realizada análise temática onde identificamos duas categorias relacionadas à satisfação no trabalho dos profissionais bancários: 1) A satisfação 
no trabalho de profissionais do setor bancário e seus desdobramentos; 2) Os fatores determinantes da satisfação no trabalho - o papel da gerência dos serviços.

\section{DISCUSSÃO}

\subsection{A satisfação no trabalho de profissionais do setor bancário e seus desdobramentos}

Analisando os estudos observa-se que a satisfação no trabalho pode atingir os indivíduos de formas diversas, podendo estar associada a vários fatores profissionais e/ou individuais, como idade, sexo, tipo de vínculo com a instituição e outros. A satisfação no trabalho pode ter variáveis antecedentes, tais como: escolaridade, excesso de trabalho, treinamentos realizados, autonomia, interação, apoio social e gênero. Há, também, fatores que são consequentes da satisfação no trabalho, como: capacidade para o trabalho, comprometimento organizacional e rotatividade (MOURÃO; MONTEIRO; VIANA, 2014).

Trabalhadores de instituições financeiras com idade mais avançada estão mais satisfeitos com a natureza do trabalho em si, sem requerer muitas mudanças ou transformações, quando comparados com trabalhadores mais jovens. Estes, por sua vez, parecem encarar seu trabalho como algo desafiador (HASSAN et al., 2011; HLATYWAYO; MHLANGA; ZINGWE, 2013). É importante dizer que, nos estudos selecionados, houve predominância da faixa etária de 20 a 29 anos entre os funcionários bancários (HASSAN et al., 2011).

Funcionários que exercem as mesmas atividades repetidamente tendem a estar mais insatisfeitos do que aqueles que possuem diversidade de tarefas (HLATYWAYO; MHLANGA; ZINGWE, 2013; OLIVIER; PERES; BEHR, 2011). Os funcionários tendem a responder positivamente ao trabalho complexo e desafiador quando eles estão satisfeitos com o seu trabalho, mais do que quando estão insatisfeitos (PIYALI et al., 2015). Isto demonstra a necessidade de os gestores identificarem e programarem estratégias que motivem os trabalhadores no seu dia-a-dia, aprimorando conhecimentos e habilidades, com ênfase no trabalho em equipe. Sentimentos gratificantes podem advir de um bom relacionamento com os colegas de trabalho, bem como da colaboração, amizade e confiança demonstrados no trabalho (PAIXÃO; BARBOSA, 2016).

O tipo de vínculo que o trabalhador possui é outro aspecto que deve ser considerado nos índices de satisfação dos profissionais. Empregados com vínculo permanente geralmente estão mais satisfeitos do que funcionários contratados temporariamente. Isto parece estar relacionado ao fato da garantia de manutenção do emprego, especialmente quando as taxas de desemprego estão em alta (FATIMAH et al., 2012; HLATYWAYO; MHLANGA; ZINGWE, 2013).

Os estudos também revelaram que indivíduos do sexo masculino apresentaram níveis mais positivos de satisfação (HLATYWAYO; MHLANGA; ZINGWE, 2013); (TLAISS, 2013). Mulheres em cargos de gestão apresentaram insatisfação com o salário, as promoções e as expectativas de progresso na carreira, se comparadas aos homens (HASSAN et al., 2011; TLAISS, 2013). É evidenciado que os homens ainda têm a preferência em ações de recrutamento para os postos de trabalho nos bancos, bem como são oferecidas a eles maiores oportunidades de promoções. Com isso, as mulheres têm ocupado menos de $20 \%$ dos cargos nesse setor (TLAISS, 2013).

Outro aspecto que deve ser destacado é que os indivíduos que apresentam capacitação, ou se mostram mais competentes, são mais propensos a se esforçar para obter resultados, estabelecer bom relacionamento com os líderes, possuir maior capacidade de enfrentar 
desafios e, consequentemente, se sentem mais reconhecidos, respeitados e mais satisfeitos com o seu trabalho (BITMIS; ERGENELI, 2011).

Ainda, os estudos revelam que há maior satisfação no trabalho entre os funcionários que ocupam cargos de gestão, visto que estão mais envolvidos com o planejamento e implementação de mudanças organizacionais, recebem maior remuneração e seus postos de trabalho possibilitam mais flexibilidade e variedade do que aqueles que não são cargos de gestão. (HLATYWAYO; MHLANGA; ZINGWE，2013; SANTOS; MOURÃO, 2011; TLAISS, 2013).

Os gestores que têm autoconfiança e habilidades para resolver problemas, permanecem com uma atitude mais positiva ao enfrentarem dificuldades, pensarem em soluções, programarem e alocarem recursos e tempo. Estes, por sua vez, se mostram mais satisfeitos com seu trabalho, além de serem eficazes em motivar a equipe para maior desempenho (KAI; YUYANG, 2011).

Acredita-se que o investimento na formação de bons líderes deve repercutir em um clima de trabalho adequado, contribuindo positivamente para o estado de ânimo da equipe. Se o indivíduo está satisfeito com seu trabalho, salário, promoções e colegas, perceberá o clima organizacional como favorável para si mesmo (BHUTTO et al., 2012).

A satisfação no trabalho deve trazer consequências não somente para o profissional, mas também para a organização. Índices de satisfação no trabalho dos profissionais bancários têm relação direta com seu desempenho profissional e comprometimento com a organização (HASSAN et al., 2011; OLIVIER; PERES; BEHR, 2011; TLAISS, 2013; GAGANDEEP, 2015). Sabe-se que a satisfação pode afetar também o aumento da produtividade, a estabilidade emocional, a disposição para aprendizagem e capacitação, o relacionamento com a liderança e a satisfação dos clientes (XIAOYAN; PUXI, 2010; YAVAS; BABACUS, 2010; ARMUTLULU; NOYAN, 2011; BITMIS; ERGENELI, 2011; BHUTTO et al., 2012; FATIMAH et al., 2012; REDDY; GUMMADI, 2013; SARAVANI; ABBASI, 2013; GEORGE; ZAKKARIYA, 2015). Assim, gestores devem ficar atentos não somente em atrair clientes, mas em promover a satisfação dos seus trabalhadores.

Com um cenário econômico sujeito a pressão e competitividade, os gestores se preocupam em controlar e reduzir custos, como forma de melhorar a competitividade e sustentar a rentabilidade organizacional (HASSAN et al., 2011; GOUNARIS; BOUKIS, 2013). No entanto, as políticas de redução de tais custos podem resultar em menores níveis de satisfação no trabalho. Se o funcionário está satisfeito, poderá executar de forma eficaz seu trabalho, por meio de um atendimento com qualidade e promovendo a melhoria da capacidade da empresa em manter ou até mesmo ampliar a sua base de clientes, pois estes estarão mais satisfeitos graças ao atendimento prestado (GOUNARIS; BOUKIS, 2013; YAVAS; BABACUS, 2010).

O investimento das organizações na capacitação para a carreira, na ampliação de oportunidades, na melhoria do clima e das condições de trabalho, bem como uma remuneração justa, de acordo com o mercado, são ferramentas que devem ser consideradas como um investimento estratégico, visando o aumento da satisfação dos trabalhadores e o alcance dos resultados almejados pela empresa, considerado como uma variável competitiva sustentável no mercado (GOUNARIS; BOUKIS, 2013).

A satisfação no trabalho melhora significativamente o desempenho e a eficácia, apoiando em longo prazo a prosperidade da instituição bancária (RIGOPOULOU et al., 2012; GAGANDEEP, 2015). Ajustes entre as habilidades e a personalidade do empregado, com os requisitos do trabalho, levam a melhores resultados, fidelidade e satisfação dos clientes, além de estimular o lucro e o crescimento da organização (YAVAS; BABACUS, 2010). 
Nesse sentido, o trabalhador satisfeito com seu trabalho pode tornar-se uma pessoa com maior possibilidade de ser um cidadão integrado à sociedade e à sua família, bem como apresentar melhores índices de bem-estar físico e emocional. Vínculos salubres no trabalho são relevantes para a sustentabilidade de uma vida saudável nos ambientes familiar e social. Ao contrário, quando há insatisfação com promoções e salário, pode haver maior rotatividade, baixa produtividade e desempenho, menor comprometimento, aumento do estresse e de problemas de saúde (FATIMAH et al., 2012; TLAISS, 2013; XIAOYAN; PUXI, 2010).

Os trabalhadores são a vantagem competitiva de qualquer organização, impactando diretamente a sua produtividade e resultados. A falta de satisfação no trabalho também resulta em taxas elevadas de absenteísmo, baixa produtividade e falta de comprometimento organizacional (GEORGE; ZAKKARIYA, 2015). Sabe-se que os fatores relacionados à satisfação no trabalho e comportamento dos funcionários, bem como as suas implicações são importantes para mensurar e posteriormente auxiliar nas decisões estratégicas da organização (MAHMUD; HASSAN; ASIF, 2014).

É possível dizer que as reações e percepções de cada profissional quanto à satisfação no trabalho são únicas, específicas a cada situação e podem afetar uns aos outros no trabalho. De fato, pesquisadores revelam que a relação entre satisfação no trabalho e comprometimento organizacional varia consideravelmente entre agências bancárias, além de variação do nível individual de satisfação (ARMUTLULU; NOYAN, 2011). Se os funcionários não estão satisfeitos com a cultura de trabalho do banco, o negócio poderá não gerar lucro. Por essa razão, a percepção do funcionário no contexto do ambiente bancário precisa ser explorada (GAGANDEEP, 2015).

\subsection{Fatores determinantes da satisfação no trabalho - o papel da gerência dos serviços}

O administrador possui o papel de influenciar a atitude dos outros gerentes e demais colegas, por meio do estabelecimento de políticas organizacionais claras e mecanismos de comunicação mais eficazes da estratégia organizacional, com o intuito de proporcionar maior satisfação no emprego (RIGOPOULOU et al., 2012; XIAOYAN; PUXI, 2010).

É possível dizer que existe uma relação significativa entre o comprometimento dos empregados, satisfação no trabalho, responsabilidade social corporativa e o estilo de liderança e práticas de trabalho (SHAFIQUR; DEBBIE; MEHRDOKHT, 2016). A liderança estratégica e transformacional, ferramenta da gerência dos serviços, deve fornecer apoio emocional, capacitação psicológica, incentivo e suporte informacional (ARMUTLULU; NOYAN, 2011; BITMIS; ERGENELI, 2011). Essa liderança deve promover o comprometimento organizacional dos trabalhadores e a satisfação no trabalho (SANTOS; MOURÃO, 2011; YAVAS; BABACUS, 2010).

Os gestores são os maiores responsáveis por difundir valores organizacionais, tais como: respeito, excelência no relacionamento com o cliente, trabalho em equipe e proatividade na gestão de riscos. Uma relação positiva com o superior, havendo delegação de responsabilidades e tarefas, otimizando as habilidades do trabalhador, também contribui para a satisfação no trabalho (MYINT et al., 2016).

Uma gestão proativa deve instituir programas de treinamento, capacitação e desenvolvimento. $\mathrm{O}$ gestor deve fornecer orientações estruturadas sobre as normas e objetivos a serem atingidos, e suporte necessário para contribuir com o desenvolvimento profissional dos funcionários, resultando em maior satisfação no trabalho e alcance dos resultados esperados (BITMIS; ERGENELI, 2011; REDDY; GUMMADI, 2013; SANTOS; MOURÃO, 
2011; SARAVANI; ABBASI, 2013; YAVAS; BABACUS, 2010; MOURÃO; MONTEIRO; VIANA, 2014; MYINT et al., 2016).

O planejamento estratégico, aliado a políticas de desenvolvimento humano e um programa de incentivos, produzirão efeitos positivos e significativos na satisfação com o trabalho, podendo resultar em baixa rotatividade, diminuição do absenteísmo e do abandono de emprego, promovendo a tendência a aprender e participar, a estar mais comprometido com as metas e a buscar a remuneração baseada no desempenho (HASSAN et al., 2011; SARAVANI; ABBASI, 2013).

Isso destaca a necessidade de um contexto de trabalho favorável para maximizar os benefícios do emprego, principalmente no setor bancário, onde a retenção de talentos é um grande desafio. A intenção do trabalhador em deixar seu posto de trabalho pode ocorrer devido a natureza, conteúdo ou projeto de trabalho, ou de outros fatores tais como remuneração, relacionamento com o supervisor ou com os colegas de trabalho, além de fatores individuais como a personalidade (PIYALI et al., 2015). Para contribuir com esse planejamento estratégico, a área de gestão de pessoas poderia desenvolver e aplicar instrumentos que avaliem a satisfação no trabalho, periodicamente e, a partir dos resultados, desenvolver novas políticas ou ações que promovam essa satisfação.

Alguns fatores que são estimuladores da satisfação no trabalho relacionados à gerência dos serviços incluem: um ambiente de trabalho saudável e educacional, inteligência emocional, suporte para o trabalho, níveis justos de remuneração e recompensas, promoções, sensação de proteção e segurança no emprego, empoderamento psicológico e liberdade para tomada de decisão, envolvimento e compromisso com o trabalho (ARMUTLULU; NOYAN, 2011; BITMIS; ERGENELI, 2011; BHUTTO et al., 2012; FATIMAH et al., 2012; HASSAN et al., 2011; HLATYWAYO; MHLANGA; ZINGWE, 2013; REDDY; GUMMADI, 2013; SARAVANI; ABBASI, 2013; MAHMUD; HASSAN; ASIF, 2014; SHAMIMHOSSAIN, 2014; MYINT et al., 2016; PAIXÃO; BARBOSA, 2016).

Podem também contribuir para a satisfação, o investimento no desenvolvimento das capacidades profissionais dos funcionários e na melhor formação da equipe, promovendo um trabalho mais flexível e desafiador com melhores níveis de salário (XIAOYAN; PUXI, 2010). Atividades recreacionais para aliviar a sobrecarga de trabalho e o investimento em um ambiente seguro também estimularão a satisfação no emprego e são responsabilidades diretas dos gestores (FATIMAH et al., 2012; HASSAN et al., 2011).

O funcionário que não se sente protegido no ambiente de trabalho, não estará satisfeito e se sentirá ameaçado pela pressão econômica, desenvolvendo sentimentos de incerteza, insegurança afetando negativamente sua satisfação, criando a intenção de rotatividade no emprego (FATIMAH et al., 2012).

Além disso, a satisfação do funcionário pode influenciar a satisfação do cliente e os resultados da empresa, traduzidos sob a forma de lucro e crescimento. Deste modo, é importante que os funcionários que se encontram em posições de linha de frente, recebam apoio e tenham seu comprometimento organizacional fortalecido (YAVAS; BABACUS, 2010). O funcionário comprometido com seu trabalho demonstrará um sentimento maior de pertencimento à organização e a recomendará para futuros funcionários e clientes.

Analisando os estudos, verificou-se que quando há predominância da insatisfação com o trabalho, esta se relaciona com: função exercida, volume do serviço, falta de comunicação entre a equipe, o relacionamento ruim entre colegas e com os líderes, pressão para alcance de metas, passando a ser uma fonte constante de estresse e em alguns casos prejuízos à saúde do funcionário, na sua qualidade de vida e no clima organizacional resultando até mesmo em 
casos de afastamento ou de suicídio (OLIVIER; PERES; BEHR, 2011; PAIXÃO; BARBOSA, 2016).

De acordo com alguns autores observa-se uma estreita relação entre a autoavaliação de saúde com a mortalidade e a morbidade do trabalhador. Assim, uma melhor compreensão dos fatores relacionados à saúde do trabalhador e à satisfação no trabalho pode indicar a necessidade do desenvolvimento de ações preventivas, de modo a manter ou a melhorar a saúde, em especial de setores submetidos a intensas reestruturações produtivas potencialmente prejudiciais ao indivíduo, como no caso de trabalhadores do setor bancário (PETRALI et al., 2015).

Segundo alguns autores, empresas que investem em Responsabilidade Social corporativa alcançam bons resultados e melhoria no desempenho financeiro, maior envolvimento dos funcionários e atração de talentos, além de construir boa reputação, aumentando a sua atratividade sob os pontos de vista social (bem-estar dos trabalhadores) e ambientalmente sustentável (SHAFIQUR; DEBBIE; MEHRDOKHT, 2016). Ao reconhecer o apoio de seu empregador para causas sociais e ambientais, os trabalhadores são inspirados para melhorar as suas atitudes no local de trabalho. Por isso os gestores possuem papel fundamental em estimular seus empregados, promovendo seu envolvimento, o que somente um local de trabalho satisfatório possibilita (KINICKI; KREITNER, 2006). A gerência deve auxiliar seus trabalhadores no reconhecimento do seu estado físico e mental, e assim contribuir para a qualidade de vida no trabalho.

A integração social, a confiança no grupo, o auxílio na execução de tarefas advindo de colegas e superiores, identificado em situações de elevado apoio social, poderiam proteger dos efeitos negativos do desgaste no trabalho sobre a saúde. O modelo atual de economia resulta, muitas vezes, em condições prejudiciais à saúde do trabalhador, afetada por desconfiança interpessoal, debilitação das relações humanas, ferramentas de avaliações de desempenho individuais mal utilizadas e políticas de gestão por metas cada vez maiores. Esses são fatores que podem contribuir para a redução da solidariedade, para a exacerbação do individualismo e para o aumento da competição no ambiente de trabalho (PETRARLI et al., 2015).

\section{CONSIDERAÇÕES FINAIS}

O setor bancário, caracterizado por fatores como competitividade e produtividade, foi fortemente afetado após a incorporação de inovações tecnológicas e organizacionais, demandando um novo ritmo de trabalho e maior necessidade de mão-de-obra qualificada. Assim, o objetivo dessa pesquisa foi, por meio da revisão integrativa da literatura, identificar a existência ou não de satisfação no trabalho bancário e quais os fatores gerenciais que interferem nessa satisfação. Percebe-se, pelos resultados, que houve avanços teóricos e metodológicos nesse campo, com o aumento das pesquisas voltadas aos trabalhadores bancários nos últimos anos, em especial no nosso país, onde encontramos poucos artigos relacionados a essa temática nos anos iniciais abrangidos nessa pesquisa.

Conclui-se pelos estudos analisados que muitos aspectos podem influenciar a satisfação dos trabalhadores de instituições financeiras, tais como: a idade do empregado, o gênero, o vínculo com a empresa, o investimento realizado em capacitação profissional, o tipo de cargo ocupado e o tipo de liderança. As consequências desta satisfação são muitas: melhores atitudes para com o trabalho, comprometimento do funcionário com a empresa, 
aumento da eficiência e da produtividade, melhor relacionamento com os colegas, e comportamentos mais éticos, contribuindo para um resultado positivo da empresa.

A gestão participativa, com tomada de decisão integrada; processos transparentes de ascensão profissional, com equidade de gênero, buscando aliar as habilidades e personalidades às necessidades do cargo, bem como um programa de incentivos e benefícios; e a promoção de padrões de comportamento profissional e ético, favorecem um bom clima de trabalho e influenciam positivamente na satisfação no emprego. Aprimorar relacionamentos, com uma comunicação clara, direcionada aos objetivos organizacionais a serem alcançados; estimular a busca de referência junto ao líder da equipe, que deve ser apoiador e incentivador; bem como recompensar o trabalho efetuado, podem contribuir para aumentar a satisfação com o trabalho dos profissionais bancários.

Nesse sentido, esta pesquisa pode contribuir em provocar a reflexão dos profissionais do setor financeiro, em especial dos gestores que devem utilizar estratégias para manter a satisfação no trabalho de sua equipe, como por exemplo, a capacitação dos profissionais. Organizações que investem em benefícios educacionais e valorizam o aprendizado contínuo destacam-se como diferenciadas nas escolhas e permanência de seus funcionários.

Este estudo apresenta como limitação, a pouca quantidade de artigos nacionais encontrados abordando essa temática, visto que mais de $80 \%$ dos resultados foram extraídos de pesquisas internacionais. Isso pode indicar a necessidade de ampliar futuros estudos, especificamente com profissionais do setor bancário de nosso país. É fundamental que gestores organizacionais compreendam o valor de manter funcionários satisfeitos a longo prazo. Esforços contínuos em futuros estudos, a fim de monitorar e avaliar níveis de satisfação, podem ser úteis na identificação de necessidades e posterior implementação de políticas ou estratégias organizacionais que promovam a satisfação no trabalho.

Assim, identificar a presença de satisfação no trabalho desses profissionais e o papel da gerência para essa satisfação deverá contribuir para o desenvolvimento de políticas organizacionais que melhorem o desempenho do empregado, com consequente alcance dos resultados esperados pela empresa. Sabemos da limitação que esse tipo de pesquisa traz, porém os autores consideram que a temática de satisfação no trabalho se constitui de extrema relevância para profissionais que atuam nesta área e, assim, pesquisas de campo devem ser conduzidas no sentido de explorar com maior profundidade o tema. 


\section{REFERÊNCIAS}

ARMUTLULU, I. H.; NOYAN. F. A multilevel model of organizational commitment. Procedia Social and Behavioral Sciences, v. 30, n. 1, p. 2139 - 2143, 2011.

BARRETO, A. C. C. Judicialização dos acidentes de trabalho: direito, necessidade ou privilégio: uma análise sócio-jurídica da sua efetividade no setor bancário. Tese (Mestrado em Políticas Sociais) - Universidade Estadual do Norte Fluminense Darcy Ribeiro, Centro de Ciências do Homem, 2015. Disponível em: <http://www.aninter.com.br/Anais\%20Coninter\%204/GT\%2022/02.\%20UMA\%20ANALISE $\% 20$ SOCIO-

JURIDICA\%20SOBRE\%20A\%20JUDICIALIZACAO\%20NOS\%20ACIDENTES\%20DE\% 20TRABALHO.pdf >. Acesso em: 08 mar. 2016.

BHUTTO, N. A. et al. A comparative study of organizational climate and job satisfaction in public, private and foreign banks. Canadian Center of Science and Education, v. 8, n. 4, p. 259-267, 2012.

BITMIS, M. G.; ERGENELI, A. Contingency approach to strategic management: a test of the mediating effect of leader member exchange on the relationship between psychological empowerment and job satisfaction in 21 st century workplace. Procedia Social and Behavioral Sciences, v. 24, n. 1, p. 1143-1153, 2011.

CURA, M. L. A. D.; RODRIGUES, A. R. F. Satisfação profissional do enfermeiro. Revista Latino-Americana de Enfermagem, 7, n. 4, p. 21-28, 1999.

ESTIVALETE, V. F. B.; ANDRADE, T. A influência dos valores organizacionais na percepção de suporte organizacional com base na concepção dos trabalhadores do setor bancário. Revista de Administração, v. 13, n. 3, p. 214-244, 2012.

FATIMAH, O. et al. Employment security as moderator on the effect of job security on worker's job satisfaction and well being. Canadian Center of Science and Education, v. 8, n. 9 , p. 50-56, 2012.

FLETCHER, J. M.; SINDELAR, J. L.; YAMAGUCHI, S. Cumulative effects of job characteristics. Health Economic, Chichester, v. 20, n. 5, p. 553-570, 2011. Disponível em: <http://www.ncbi.nlm.nih.gov/pmc/articles/PMC3066461/pdf/nihms212769.pdf>. Acesso em 17 jun. 2016.

GAGANDEEP, K. Perception of bank employees' towards working environment of selected Indian universal banks. International Journal of Bank Marketing, v. 33, n.1, p. 58-77. 2015. Disponível em: <http://www.emeraldinsight.com/doi/pdfplus/10.1108/IJBM-10-20130117>. Acesso em: 26 jan. 2016.

GEORGE, E.; ZAKKARIYA, K. A. Job related stress and job satisfaction: a comparative study among bank employees. Journal of Management Development, v. 34, n. 3, p. 316329. 2015. Disponível em: <http://www.emeraldinsight.com/doi/pdfplus/10.1108/JMD-072013-0097>. Acesso em: 26 jan. 2016. 
GIRONDI, S. T. Satisfação, motivação e perspectivas dos bancários que assumiram suas funções no Banco do Brasil S.A, a partir do ano de 1998, no estado do Rio Grande do Sul. Especialização em gestão de negócios financeiros. Universidade Federal do Rio Grade do Sul - Escola de Administração, 2007 Disponível em: <http://www.lume.ufrgs.br/bitstream/handle/10183/14204/000649778.pdf?sequence=1>. Acesso em 01 mar. 2016.

GOUNARIS, S.; BOUKIS, A. The role of employee job satisfaction in strengthening customer repurchase intentions. Journal of Services Marketing, v. 27, n. 4, p. 322-333, 2013.

HAREEM, H. Human Resource management, framework integrated. Amman: Hamed Publishing House and Distribution, 2013.

HASSAN, Y. et al. Job satisfaction in private banking sector of Pakistan. Global Journal of Management and Business Research, v. 11, n. 12, p. 84-94, 2011.

HLATYWAYO, C. K.; MHLANGA, T. S.; ZINGWE, T. Neuroticism as a determinant of job satisfaction among bank employees. Mediterranean Journal of Social Sciences, v. 4, n. 13, p. 549-554, 2013.

KAI Y.; YUYANG, W. Research on the relations between managerial self-efficacy and job satisfaction. 2011. IEEE Xplore Digital Library. Acess provided by Universidade de São Paulo. Disponível em: $<$ http://ieeexplore.ieee.org/stamp/stamp.jsp?tp=\&arnumber=5997995\&tag=1 >. Acesso em: 14 jun. 2015.

KAUR, G. Perception of bank employees' towards working environment of selected Indian universal banks. International Journal of Bank Marketing, v. 33, n.1, p. 58-77, 2015. Disponível em: <http://www.emeraldinsight.com/doi/pdfplus/10.1108/IJBM-10-2013-0117>. Acesso em: 26 jan. 2016.

KINICKI, A. I.; KREITNER, R. Comportamento organizacional. São Paulo: McGraw-Hill, 2006.

LOCKE, E. A. What is job satisfaction?. Organization Behavior and Human Performance, v. 4, n. 4, p. 309-336, 1969.

LOCKE, E. A. The nature and causes of job satisfaction. In: DUNNETE, M. D. (ed.) Handbook of industrial and organizational psychology. Chicago: Rand McNally College Publishing, 1976.

LUNARDI FILHO, W. D. Prazer e sofrimento no trabalho: contribuições à organização do processo de trabalho da enfermagem. Tese (Mestrado em Administração) - Faculdade de Ciências Econômicas, Universidade Federal do Rio Grande do Sul, 1995.

MACHADO, M. M.; JAROUJ, C. A. Pesquisa de clima organizacional em uma instituição financeira de Blumenau SC. Revista Interdisciplinar Científica Aplicada, v. 1, n. 4, p. 01- 
22, 2007. Disponível em: <http://rica.unibes.com.br/index.php/rica/article/view/65/61>. Acesso em: 12 mai.2016.

MAHMUD, M. T.; HASAN, M. T.; ASIF, A. S. M. Determinants of Job Satisfaction: A Comparative Study in the Banking Sector of Bangladesh. International Journal of Economics and Empirical Research, v. 2, n.1, p. 22-28, 2014. Disponível em: <http://tesdo.org/shared/upload/pdf/papers/IJEER,\%202_1_22-28.pdf>. Acesso em: 31 mai.2016

MAXIMO, T. A. C. O. et al. Exigências nos percursos profissionais de gerentes de bancos. Psicologia \& Sociedade, v. 23, n. 1, p. 66-74, 2011.

MENDES, K. D. S.; SILVEIRA, R. C. C. P.; GALVÃO, C. M. Revisão integrativa: método de pesquisa para a incorporação de evidências na saúde e na enfermagem. Texto \& Contexto-Enfermagem, v. 17, n. 4, p. 758-764, 2008.

MINAYO, M. C. S.; ASSIS, S. G.; OLIVEIRA, R. V. C. Impacto das atividades profissionais na saúde física e mental dos policiais civis e militares do Rio de Janeiro. Ciência \& Saúde Coletiva, v. 16, n. 4, p. 2199-2209, 2011.

MOURÃO, L., MONTEIRO, A. C. F., VIANA, V. R. A influência do desenvolvimento profissional e da identificação organizacional na satisfação no trabalho. Psico, v. 45, n. 2, pp. 198-208, 2014. Disponível em: <http://revistaseletronicas.pucrs.br/ojs/index.php/revistapsico/article/view/13470/11709>. Acesso em: 30 jun. 2016.

MYINT, S. S. et al. An analysis of employee satisfaction of private banks in Myanmar. International Business Management, v. 10, n.1, p. 101-114, 2016. Disponível em: $<$ http://docsdrive.com/pdfs/medwelljournals/ibm/2016/101-114.pdf>. Acesso em: 31 mai. 2016.

NAVARRO, V. L.; CARRIJO, D. C. M. Ler e planos de demissão voluntária: trajetórias de dor e sofrimento entre bancários. Cadernos de Psicologia Social do Trabalho, v. 12, n. 1, p. 157-171, 2009. Disponível em: 〈http://pepsic.bvsalud.org/pdf/cpst/v12n2/a03v12n2.pdf>. Acesso em 10 fev. 2016.

NEVES, M. Y. R.; SELIGMANN, E. S.; A dor e a delícia de ser (estar) professora: trabalho docente e saúde mental. Estudos e Pesquisas em Psicologia, v. 6, n. 1, p.63-65, 2006.

OLIVIER, M.; PEREZ, C. S.; BEHR, S. C. F. Trabalhadores afastados por transtornos mentais e de comportamento: o retorno ao ambiente de trabalho e suas consequências na vida laboral e pessoal de alguns bancários. Revista de Administração Contemporânea, v. 15, n. 6, p.993-1015, 2011.

PAIXÃO, A. R.; BARBOSA, M. A. C. Satisfação no trabalho: um estudo na agência da caixa econômica federal de Cícero Dantas - BA. Id on Line Revista Multidisciplinar e de Psicologia, v. 10, n. 30, p. 10-26, 2016. Disponível em: <https://idonline.emnuvens.com.br/id/article/view/428/534>. Acesso em: 30 jun. 2016. 
PETARLI, G. B. et al. Autoavaliação do estado de saúde e fatores associados: um estudo em trabalhadores bancários. Cad. Saúde Pública, v. 31, n. 4, p. 787-799, 2015. Disponível em: $<$ http://www.scielo.br/pdf/csp/v31n4/0102-311X-csp-31-04-00787.pdf>. Acesso em 30 mai. 2016.

PIYALI, G. et al. Exploring the moderating role of context satisfaction between job characteristics and turnover intention of employees of Indian public sector Banks. Journal of Management Development, v. 34, Iss 8, p. 1019 - 1030, 2015. Disponível em: < http://dx.doi.org/10.1108/JMD-10-2014-0138 >. Acesso: 26 jan. 2016.

REDDY, A. V.; GUMMADI, A. An empirical study on the relationship between determinants of job satisfaction and job satisfaction itself among the employeers of both public and private sector banks of Guntur Urban Region. Internacional Business Management - Medwell Journals, v. 7, n. 6, p. 446-451, 2013

RIGOPOULOU, I. et al. Information control, role perceptions, and work outcomes of boundary-spanning frontline managers . Journal of Business Research, v. 65, n. 1, p. 626633, 2012.

RUEDA, F. J. M. Análise fatorial confirmatória da escala de satisfação no trabalho nas versões de 25 e 15 itens. Revista Psicologia: Organizações e Trabalho, v. 15, n. 1, p. 82-88, 2015. Disponível em: <http://pepsic.bvsalud.org/pdf/rpot/v15n1/v15n1a08.pdf>. Acesso em 25 fev. 2016.

SANTOS, J. R. V. S.; MOURÃO, L. Impacto do treinamento como variável preditora da satisfação com o trabalho. Revista de Administração, v. 46, n. 3, p. 305-318, 2011.

SARAVANI, S. R.; ABBASI, B. Investigating the influence of job rotation on performance by considering skill variation and job satisfaction of bank employees. Technical Gazette, v. 20, n. 3, p. 473-478, 2013.

SEVERINO, M. R.; EID, F.; CHIARELLO, C. L. Organização na economia solidária desafios e limites na construção de modelo alternativo ao Taylorismo. Revista Pegada, v. 14, n. 2, p. 143-162, 2013.

SHAFIQUR, R; DEBBIE, H-L; MEHRDOKHT, P. The effect of employee CSR attitudes on job satisfaction and organizational commitment: evidence from the Bangladeshi banking industry. Social Responsibility Journal, v. 12, n. 2, p. 228-246, 2016.

SHAMIMHOSSAIN, M. D. Job satisfaction of bank employees in Bangladesh. Research Journal of Finance and Accounting, v. 5, n. 1, p. 1-8, 2014. Disponível em: <http://www.iiste.org/Journals/index.php/RJFA/article/view/10448/10641>. Acesso em 01 ago. 2015.

SILVA, J. L.; NAVARRO, V. L. Organização do trabalho e saúde dos trabalhadores bancários. Revista Latino-Americana de Enfermagem, v. 20, n. 2, p. 226-234, 2012.

SILVA, L. S.; BARRETO, S. M. Stressful working conditions and poor self-rated health among financial services employees. Revista de Saúde Pública, v. 46, n. 3, p. 407-416, 
2012. Disponível em: <http://www.scielosp.org/pdf/rsp/v46n3/3520.pdf >. Acesso em: 26 jan. 2016.

SILVA, P. S.; LIMA, S. M. O. Avaliação da qualidade de vida no trabalho dos colaboradores do Banco do Brasil S/A da agência Borborema - Campina Grande - PB. Qualitas Revista

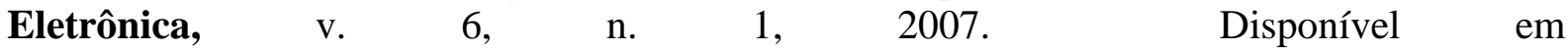
<http://revista.uepb.edu.br/index.php/qualitas/article/view/97/65>. Acesso em: 01 mar.2016.

SIQUEIRA, M. M. M. (Org.). Medidas do comportamento organizacional: ferramentas de diagnóstico e de gestão. Artmed: Porto Alegre, 2008.

SOUZA, M. T.; SILVA, M. D.; CARVALHO, R. Revisão integrativa: o que é e como fazer. Einstein, $8(1 \quad \mathrm{Pt} \quad 1), \quad$ p.102-6, $2010 . \quad$ Disponível em: <http://www.scielo.br/pdf/eins/v8n1/pt_1679-4508-eins-8-1-0102>. Acesso em: 17 jun. 2016

TLAISS, H. A. Determinants of job satisfaction in the banking sector: the case of Lebanese managers. Employee Relations, v. 35, n. 4, p. 377-395, 2013. Disponível em <www.emeraldinsight.com/0142-5455.htm>. Acesso em: 22 ago. 2015.

VALLE, A. R. Monitoramento da satisfação no trabalho em uma Empresa Financeira. Brasília, 2007. Tese (Doutorado em Psicologia) - Universidade de Brasília UNB, 2007.

WHITTEMORE, R.; KNALF, K. The integrative review: update methodology. Journal of Advanced Nursing, v. 52, n. 5, p. 5546-53, 2005.

XIAOYAN, Z.; PUXI, O. Comparison of factors influencing job satisfaction in domestic and foreign banks in China. IEEE Xplore Digital Library. Acess provided by Universidade de São $\quad$ Paulo. 2010. Disponível em: <http://ieeexplore.ieee.org/stamp/stamp.jsp?tp=\&arnumber=5691854>. Acesso em: 14 jun. 2015.

YAVAS, U.; BABACUS, E. Relationships between organizational support, customer orientation, and work outcomes A study of frontline bank employees. International Journal of Bank Marketing, v. 28, n. 3, p. 222-238, 2010. Disponível em: <www.emeraldinsight.com/0265-2323.htm>. Acesso em: 10 ago. 2015. 